\title{
Research on the Construction of Product Material Perceptual Evaluation Model Based on EEG Physiological Cognition
}

\author{
Xudong Wang and Li Lin* \\ School of Mechanical Engineering, Guizhou University, Guiyang, Guizhou, 550025, China
}

\begin{abstract}
In order to improve the user experience of industrial product materials and solve the problems of strong subjectivity and lack of objective data support in the evaluation of industrial product materials, a perceptual evaluation method of product materials based on EEG is proposed, and a comprehensive model of perceptual evaluation of industrial product materials combining psychological and physiological evaluation indexes is established. 20 subjects were recruited to carry out perceptual evaluation tests on 8 kinds of teacups made of different materials, and the established model was calculated mathematically to select the optimal material scheme. The experimental results verified the effectiveness of the model, indicating that the psychological and physiological indexes of users can be mutually verified in the perceptual evaluation of product materials.
\end{abstract}

\section{Introduction}

With the rapid development of science and technology, increasingly fierce market competition and diversified consumer demand, the life cycle of products is also increasingly shortened [1]. In view of the gradual improvement of people's consumption level, consumers do not only consider functions when purchasing products, but also pay more attention to the visual and tactile sensory experiences brought by product modeling, color, material, etc. [2]. When evaluating the intuitive perception of industrial design renderings, consumers usually can only experience the visual perception from the surface material of the products [3]. Therefore, at the beginning of product development, the traditional product evaluation method is no longer sufficient to cope with the predicament of emotional needs of users, diversified production and shortened product life cycle [4].

In view of the above problems, this paper takes product material perception evaluation as the breakthrough point, combined with subjective psychological evaluation, deeply studies user's perceptual evaluation of product material, and constructs an evaluation model [5]. In order to solve the problem of lack of material expression, on the one hand, it provides design basis for designers, on the other hand, the products spread to the market meet users' needs and meet users' psychological expectations [6].

\section{Principle of physiological/ psychological cognitive test for product material users}

People's feelings on the evaluation of things are relatively abstract and vague [7]. This paper will mainly use the spectrum analysis method, obtain the brain wave, through the proportion of the two waveforms to explore the relationship between the product tactile perception evaluation and the product tactile perception evaluation, using event-related potential technique, extracting the changes of cerebral cortex potential caused by tactile stimulation acting on senses [8]. This paper explores the relationship between the early component $\mathrm{P} 2$ related to stimulation physical attributes and the late component P3 related to cognitive processing and the perceptual evaluation of product materials [9]. Finally, a perceptual evaluation model of product materials based on EEG physiological cognition is constructed by combining the psychological cognitive evaluation of supervisors.

\section{EEG physiological cognition-based user perceptual evaluation method for product materials}

\subsection{Construction of psychological and physiological perceptual evaluation system for product material users}

According to the research on product material evaluation in the existing literature, the characteristic dimensions that professional material evaluators can perceive include texture, smoothness, wetness, hardness, and warmth and coldness [10]. Finally, the user experience evaluation indexes of industrial design materials are determined as smoothness, hardness and texture, as shown in the figure, the 7-level Richter Scale "Extremely Dissatisfied", "Very Dissatisfied", "Dissatisfied", "General", "Satisfied", "Very Satisfied" and "Extremely Satisfied" is used to test the

*Corresponding author's e-mail: yangmq99@126.com 
user's satisfaction with different energy dimensions of the material. The higher the user's evaluation level, the greater the contribution to the overall evaluation of the material [11]. Therefore, smoothness, hardness and texture are all positive indicators.

\subsection{Comprehensive evaluation model matrix based on EEG physiological cognition}

Let the set of test schemes be $C=\left\{c_{1}, c_{2}, c_{3}, \ldots, c_{m}\right\}$ and the set of subjects be $B=\left\{b_{1}, b_{2}, b_{3}, \ldots, b_{n}\right\}$. Where: $m$ represents the number of test schemes and $n$ represents the set of subjects.

3.2.1 Psychological perceptual evaluation model for product Material users. Subject $n^{\prime}$ evaluated scheme $m^{\prime}$ as $p_{n^{\prime}, m^{\prime}}$, then subjects $\left(b_{1}, b_{2}, \ldots b_{n}\right)$ scored schemes $\left(c_{1}, c_{2}, \ldots c_{m}\right)$ in turn is as follows:

$$
P=\left[\begin{array}{cccc}
p_{1,1} & p_{1,2} & \cdots & p_{1, \mathrm{~m}} \\
p_{2,1} & p_{2,2} & \cdots & p_{2, \mathrm{~m}} \\
\vdots & \vdots & & \cdots \\
p_{n, 1} & p_{2,2} & \cdots & p_{n, \mathrm{~m}}
\end{array}\right]
$$

The combined score of the program $i$ is:

$$
p_{i}=\frac{p_{1, i}+p_{2, i}+\cdots+p_{n, i}}{n}
$$

The combined score for each program is:

$$
\boldsymbol{P}^{T}=\left[\begin{array}{lllll}
\boldsymbol{P}_{c_{1}} & \boldsymbol{P}_{c_{2}} & \ldots & \boldsymbol{P}_{c_{n}}
\end{array}\right]
$$

3.2.2 EEG test evaluation model for product material user experience. The EEG data of 32 electrodes in cerebral cortex were synchronously recorded by EEG when subjects touched and experienced different product material schemes can be recorded as:

$$
D\left(b_{i}, c_{i}, G\right)=\left[\begin{array}{cccc}
d\left(b_{1}, c_{1}, G\right) & d\left(b_{1}, c 2 G\right) & \cdots & d\left(b_{1}, c_{m}, G\right) \\
d\left(b_{2}, c_{1}, G\right) & d\left(b_{2}, c_{2}, G\right) & \cdots & d\left(b_{2}, c_{m}, G\right) \\
\vdots & \vdots & & \vdots \\
d\left(b_{n}, c_{1}, G\right) & d\left(b_{n}, c_{2}, G\right) & \cdots & d\left(b_{n}, c_{m}, G\right)
\end{array}\right]
$$

In the formula, $D\left(b_{i}, c_{j}, G\right)$ is the EEG data of the subject $b_{i}$ experience test scheme $c_{j}$ and $G=\left(g_{1}\right.$, $\left.g_{2}, \ldots g_{i}, \ldots, g_{32}\right) g_{i}$ represents the EEG data of the electrode $i$. is

The combined value of EEG data of all test schemes $j$

$$
d\left(b_{1 \sim n}, c_{j}, G\right)=\frac{d\left(1, c_{j}, G\right)+d\left(2, c_{j}, G\right)+\cdots+d\left(n, c_{j}, G\right)}{n}
$$

The combined value of EEG data of each scheme is

$$
D^{T}=\left[\begin{array}{llll}
d_{c_{1}} & d_{c_{2}} & \ldots & d_{c_{m}}
\end{array}\right]
$$

3.2.3 Comprehensive model of product material perceptual evaluation based on EEG physiological cognition combining. The subjective psychological evaluation and objective EEG evaluation data of users, a material comprehensive evaluation model based on Kansei Engineering and EEG fusion is proposed to improve the accuracy of objective evaluation of product design.

The comprehensive values of psychological evaluation $P$ and EEG data $D$ are integrated to form a fused data matrix $Z$ :

$$
Z=[P, Y, E]=\left[\begin{array}{ccc}
p_{c 1} & y_{c 1} & d_{c 1} \\
p_{c 2} & y_{c 2} & d_{c 2} \\
\vdots & \vdots & \vdots \\
p_{c m} & y_{c m} & d_{c m}
\end{array}\right]
$$

\section{Test experiment of product material sensitivity evaluation based on EEG physiological cognition}

The essence of EEG is a neuro-bioelectric activity. Potential changes accompanied by nerve activities are the reflection of human thinking, emotion, mental and psychological activities. In this experiment, the hand feeling of teacups made of different materials was taken as the research content, and the changes of cerebral cortex potential of hand-to-cup touch feeling information were recorded to obtain the time process of brain-to-cup touch information processing.

\subsection{Experimental research objects}

In the experiment, 20 graduate students (aged 19-26) majoring in design were selected. All the subjects participated voluntarily and were right-handed, and no history of neurological diseases.

\subsection{Experimental equipment}

The data were measured using EPOC Flex equipment of Emotive Company in the United States, with a sampling rate of $128 \mathrm{~Hz}$ and 32 electrode channels.

\subsection{Experimental materials}

Eight cups with the same shape and size with different material samples (ceramic, sand, glass, jade, iron, copper, silver and wood) are configured, and user perception evaluation is carried out with cup body materials.

\subsection{Experimental process}

(1) The subjects read the experimental instructions and watched the design renderings on the computer.

(2) Experiment: The subjects experienced the visual and tactile perception of the materials of several experimental samples respectively. 
(3) 20 subjects wore EEG to evaluate the material evaluation indexes (smoothness, hardness and comfort) of several experimental physical samples at the same time. The experience of the material samples was scored through keyboard numeric keys 1-5 (1-very poor, 2relatively poor, 3-general, 4-relatively good, 5-very good). At the same time of pressing the keyboard, this evaluation ends and enters the next evaluation index. Until all the material experience is completed.

(4) After the experiment, the EEG stopped recording.

\section{Data processing of product material perceptual evaluation test based on EEG physiological cognition}

\subsection{Product material perceptual evaluation user psychological scale value}

Substituting the user's subjective psychological evaluation data into Formula (1), Formula (2) and Formula (3) can obtain the comprehensive psychological values of all subjects for 8 cup material sample schemes, as shown in Table 1.

\subsection{EEG data processing for perceptual evaluation of product materials}

\subsection{Pre-treatment}

Step 1 Removes physiological interference signals. Step 2 EEG data segmentation.

Step 3 Digital Filtering.

Step 4 Average Amplitude.

Table 1. Comprehensive psychological scale for users' perceptual evaluation of material scheme

\begin{tabular}{ccccccccc}
\hline Sample of & Scheme & Scheme & Scheme & Scheme & Scheme & Scheme & Scheme & Scheme \\
teacup material & 1 & 2 & 3 & 4 & 5 & 6 & 7 & 8 \\
$\begin{array}{c}\text { Comprehensive } \\
\text { psychological } \\
\text { scale }\end{array}$ & 3.8 & 3.3 & 2.7 & 4.2 & 2.6 & 3.1 & 3.5 & 3.4 \\
\hline
\end{tabular}

\subsubsection{Analysis of evoked brain electrical activity map.} The independent component analysis module (ICA) of EEGLAB EEG data processing software is used to carry out independent component analysis on the processed EEG data, and the brain topographic maps of 32 electrode data of all subjects are drawn respectively. Therefore, the distribution of EEG energy induced by each material sample can be observed more intuitively. If the average energy of event-related potentials (ERP) in the region is larger, the darker the color of the region will be. The brain electrical activity map induced by the stimulation of material sample 7 is shown in Figure 1.

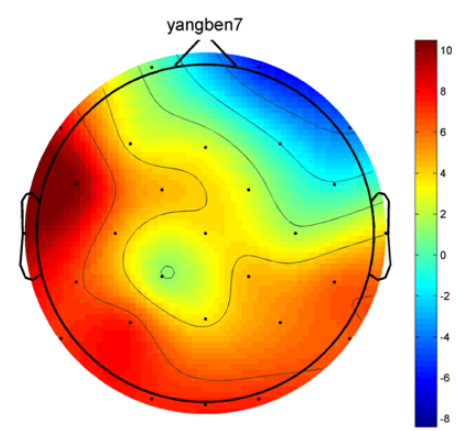

Figure 1. Evoked brain electrical activity map of material scheme 7

Combined with the electrode distribution map and energy topography map, it can be found that the active brain regions stimulated by the material sample scheme are mainly concentrated in the frontal lobe region of the brain, including FP1, FP2, F3, F4, FZ, F7, F8, FC1, FC2, FC5 and other electrodes related to aesthetic cognitive evaluation.

5.2.3 Analysis of evoked brain waveform. The electrodes related to aesthetic cognitive experience in ERP include: FP1, FP2, F3, F4, FZ, F7, F8, FC1, FC2, FC5. ERP component P300 mainly includes P300, which refers to the positive wave with the latent value of $200 \mathrm{~ms}$ $600 \mathrm{~ms}$ in ERP. When users experience 8 different material schemes, the ERP data of the above 10 electrodes will be different, so it is necessary to process, extract and analyze the data.

\subsection{Validation of evaluation model}

Substituting the psychological values and EEG values of all the subjects into the comprehensive evaluation model of product materials (Equation 7), the evaluation results of all the subjects on 8 material schemes can be obtained.

\section{Analysis of evaluation results}

\subsection{Correlation analysis}

Twenty subjects analyzed the psychological value P of 8 material schemes and the peak values of EEG FP1, FP2, F3, F4, FZ, F7, F8, FC1, FC2, FC5, and obtained the correlation coefficient analysis results, as shown in Table 2 . 
Table 2. Correlation between psychological volume and EEG

\begin{tabular}{cccccccccccc}
\hline & P & FP1 & FP2 & F3 & F4 & FZ & F7 & F8 & FC1 & FC2 & FC5 \\
\hline P & 1 & 0.93 & 0.72 & 0.89 & 0.78 & 0.96 & 0.94 & 0.86 & 0.68 & 0.78 & 0.84 \\
FP1 & 0.78 & 1 & 0.99 & 0.99 & 0.86 & 0.93 & 0.84 & 0.99 & 0.92 & 0.95 & 0.76 \\
FP2 & 0.89 & 0.98 & 1 & 0.94 & 0.84 & 0.90 & 0.97 & 0.92 & 0.95 & 0.89 & 0.93 \\
F3 & 0.87 & 0.88 & 0.97 & 1 & 0.73 & 0.97 & 0.54 & 0.84 & 0.78 & 0.56 & 0.82 \\
F4 & 0.94 & 0.91 & 0.78 & 0.91 & 1 & 0.86 & 0.73 & 0.76 & 0.81 & 0.93 & 0.94 \\
FZ & 0.97 & 0.95 & 0.90 & 0.78 & 0.89 & 1 & 0.97 & 0.89 & 0.65 & 0.91 & 0.87 \\
F7 & 0.91 & 0.83 & 0.84 & 0.67 & 0.94 & 0.94 & 1 & 0.95 & 0.98 & 0.71 & 0.99 \\
F8 & 0.96 & 0.89 & 0.93 & 0.82 & 0.99 & 0.88 & 0.77 & 1 & 0.96 & 0.83 & 0.68 \\
FC1 & 1.00 & 0.92 & 0.87 & 0.73 & 0.92 & 0.91 & 0.96 & 0.84 & 1 & 0.88 & 0.85 \\
FC2 & 0.95 & 0.87 & 0.95 & 0.89 & 0.84 & 0.91 & 0.73 & 1.00 & 0.93 & 1 & 0.89 \\
FC5 & 0.84 & 0.90 & 0.81 & 0.93 & 0.93 & 0.75 & 0.97 & 0.76 & 0.86 & 0.93 & 1 \\
\hline
\end{tabular}

From the above analysis, it can be seen that the correlation coefficients among the 11 indexes such as the psychological and physiological values of the product material sample scheme are positive, and all the other correlation coefficients are greater than 0.6 except $\mathrm{F} 3$ and F7, F3 and FC2, which are 0.54 and 0.56 respectively, thus showing good correlation.

\subsection{Relative closeness analysis}

In this evaluation, to comprehensively evaluate and make the indexes in different dimensions feasible, it is necessary to analyze the advantages and disadvantages of each scheme in different physiological and psychological dimensions. It is necessary to carry out relative closeness analysis on the comprehensive data $\mathrm{Z}$ and normalize the indexes in the value $Z$ to the [ $\left.\begin{array}{ll}0 & 1\end{array}\right]$ range. The details are as follows:

Step 1: Obtain the optimal value and the worst value of each scheme evaluation index.

The calculation process of the optimal value $F_{i j}^{+}$and the worst value $F_{i j}^{-}$of each evaluation index is as follows:

$$
\begin{aligned}
& F_{i j}^{+}=\max \left\{Z_{i j}\right\}, i=1,2, \ldots, 8 ; j=1,2, \ldots, 11 \\
& F_{i j}^{-}=\min \left\{Z_{i j}\right\}, i=1,2, \ldots, 8 ; j=1,2, \ldots, 11
\end{aligned}
$$

In the formula, $Z_{i j}$ is the value of the index of the $\mathrm{i}$-th material sample scheme in the $j$-th evaluation dimension.

Step 2: Obtains the distance from the evaluation value to the optimal value and the worst value.

$$
\begin{aligned}
& d_{i j}^{+}=F_{i j}^{+}-F_{i j} \\
& d_{i j}^{-}=F_{i j}^{+}-F_{i j}
\end{aligned}
$$

In the formula, $d_{i j}^{+}$is the distance between the $\mathrm{j}$-th evaluation index and the optimal value of the $i$-th material sample scheme, and $d_{i j}^{-}$is the distance between the $\mathrm{j}$-th evaluation index and the worst value of the $i$-th material sample scheme.

Step 3: Obtains the relative closeness of the evaluation value.

$$
\mathrm{Z}_{\mathrm{ij}}=\frac{\mathrm{d}_{\mathrm{ij}}^{-}}{\mathrm{d}_{\mathrm{ij}}^{-}+\mathrm{d}_{\mathrm{ij}}^{+}}
$$

In the formula, $Z_{i j} \in\left[\begin{array}{ll}0 & 1\end{array}\right], i=1,2, \ldots, 8, j=1,2, \ldots$,

\begin{tabular}{|c|c|c|c|c|c|c|c|c|c|}
\hline \multirow{2}{*}{$\begin{array}{l}\text { Dimension } \\
\text { indication }\end{array}$} & \multicolumn{8}{|c|}{ Sample of product material scheme } & \multirow{2}{*}{ Sort } \\
\hline & $\mathrm{C} 1$ & $\mathrm{C} 2$ & $\mathrm{C} 3$ & $\mathrm{C} 4$ & $\mathrm{C} 5$ & $\mathrm{C} 6$ & $\mathrm{C} 7$ & $\mathrm{C} 8$ & \\
\hline $\mathrm{P}$ & 0.95 & 0.77 & 0.68 & 1.00 & 0.22 & 0.00 & 0.55 & 0.27 & $\begin{aligned} \mathrm{C} 4>\mathrm{C} 1>\mathrm{C} 2 & >\mathrm{C} 3>\mathrm{C} 7>\mathrm{C} \\
8 & >\mathrm{C} 5>\mathrm{C} 6\end{aligned}$ \\
\hline FP1 & 0.84 & 0.78 & 0.72 & 1.00 & 0.00 & 0.26 & 0.64 & 0.49 & $\begin{aligned} \mathrm{C} 4>\mathrm{C} 1>\mathrm{C} 2 & >\mathrm{C} 3>\mathrm{C} 7>\mathrm{C} \\
8 & >\mathrm{C} 6>\mathrm{C} 5\end{aligned}$ \\
\hline FP2 & 0.44 & 0.37 & 0.29 & 1.00 & 0.12 & 0.21 & 0.34 & 0.23 & $\begin{aligned} \mathrm{C} 4>\mathrm{C} 1>\mathrm{C} 2 & >\mathrm{C} 7>\mathrm{C} 3>\mathrm{C} \\
8 & >\mathrm{C} 6>\mathrm{C} 5\end{aligned}$ \\
\hline F3 & 0.33 & 0.28 & 0.24 & 1.00 & 0.11 & 0.09 & 0.20 & 0.16 & $\begin{aligned} \mathrm{C} 4>\mathrm{C} 1>\mathrm{C} 2>\mathrm{C} 3>\mathrm{C} 7>\mathrm{C} \\
8>\mathrm{C} 5>\mathrm{C} 6\end{aligned}$ \\
\hline F4 & 0.31 & 0.28 & 0.20 & 1.00 & 0.03 & 0.00 & 0.15 & 0.12 & $\begin{aligned} \mathrm{C} 4>\mathrm{C} 1>\mathrm{C} 2 & >\mathrm{C} 3>\mathrm{C} 7>\mathrm{C} \\
8 & >\mathrm{C} 5>\mathrm{C} 6\end{aligned}$ \\
\hline FZ & 0.79 & 0.64 & 0.57 & 1.00 & 0.28 & 0.32 & 0.52 & 0.44 & $\begin{aligned} \mathrm{C} 4>\mathrm{C} 1>\mathrm{C} 2 & >\mathrm{C} 3>\mathrm{C} 8>\mathrm{C} \\
7>\mathrm{C} 6 & >\mathrm{C} 5\end{aligned}$ \\
\hline F7 & 0.41 & 0.33 & 0.24 & 1.00 & 0.05 & 0.00 & 0.22 & 0.13 & $\begin{aligned} \mathrm{C} 4>\mathrm{C} 1>\mathrm{C} 2 & >\mathrm{C} 3>\mathrm{C} 7>\mathrm{C} \\
8 & >\mathrm{C} 5>\mathrm{C} 6\end{aligned}$ \\
\hline F8 & 0.64 & 0.51 & 0.45 & 1.00 & 0.00 & 0.13 & 0.33 & 0.21 & $\begin{aligned} \mathrm{C} 4>\mathrm{C} 1>\mathrm{C} 2>\mathrm{C} 3>\mathrm{C} 7>\mathrm{C} \\
8>\mathrm{C} 6>\mathrm{C} 5\end{aligned}$ \\
\hline $\mathrm{FC} 1$ & 0.82 & 0.65 & 0.52 & 1.00 & 0.00 & 0.27 & 0.42 & 0.47 & $\begin{array}{c}\mathrm{C} 4>\mathrm{C} 1>\mathrm{C} 2>\mathrm{C} 3>\mathrm{C} 8>\mathrm{C} \\
7>\mathrm{C} 6>\mathrm{C} 5\end{array}$ \\
\hline $\mathrm{FC} 2$ & 0.73 & 0.47 & 0.39 & 1.00 & 0.10 & 0.00 & 0.36 & 0.29 & $\begin{aligned} \mathrm{C} 4>\mathrm{C} 1>\mathrm{C} 2>\mathrm{C} 3>\mathrm{C} 7>\mathrm{C} \\
8>\mathrm{C} 5>\mathrm{C} 6\end{aligned}$ \\
\hline FC5 & 0.40 & 0.28 & 0.24 & 1.00 & 0.07 & 0.00 & 0.21 & 0.13 & $\begin{array}{c}\mathrm{C} 4>\mathrm{C} 1>\mathrm{C} 2>\mathrm{C} 3>\mathrm{C} 7>\mathrm{C} \\
8>\mathrm{C} 5>\mathrm{C} 6\end{array}$ \\
\hline
\end{tabular}
11. Substituting the data into Equation (12), the closeness degree and ranking table of evaluation values of each material scheme are obtained, as shown in Table 3.

Table 3. Close degree and ranking table of evaluation value and optimal value 
As can be seen from the above table, after relative closeness analysis, the evaluation indexes are normalized for easy comparison. For example, on the index FC1, the 8 material sample schemes $\mathrm{C} 4$ have the highest closeness score and the best user experience, then the ranking of the 8 material schemes is: $\mathrm{C} 4>\mathrm{C} 1>\mathrm{C} 2>\mathrm{C} 3>\mathrm{C} 8>\mathrm{C} 7>$ $\mathrm{C} 6>\mathrm{C} 5$

\section{Conclusions}

This paper takes teacups as samples in the perceptual evaluation of product materials, combined with the characteristics and limitations of product material evaluation methods, a perceptual evaluation model of product material based on the combination of subjective psychological evaluation and objective psychological evaluation of EEG technology is designed. The experimental results show that there is a positive correlation between the subjective psychological values used for perceptual evaluation of product materials and the physiological indexes of EEG, and the evaluation results of each dimension can be verified by each other. The experimental results show that the method has certain universality and can be used in other fields such as scheme selection in the primary stage of industrial product design and subsequent iterative updating of products in the future.

\section{Acknowledgments}

Funding: This project was supported by the National Natural Science Foundation of China (grant numbers 51865003) and Guizhou Provincial Science and Technology Plan Project (grant numbers [2018] 5781).

\section{References}

1. Papagiannidis, S., See-To, E., \& Bourlakis, M. (2014). Virtual test-driving: The impact of simulated products on purchase intention. Journal of Retailing and Consumer Services, 21(5), 877887.

2. Zhang Lei, Ge Weimin, Li Lingling, \& Zhong Shujin. Summary of Definition, Category, Method and Development Trend of Industrial Design. Mechanical Design (8), 101-105.

3. Luo Shijian \& Zhu Shangshang. (2010). Experience and Product Innovation Design. Machinery Industry Press.

4. Yang, M.Q., Lin, L., Chen, Z.A., Wu, L., \& Guo, Z.E. (2020). Research on the construction method of kansei image prediction model based on cognition of EEG and ET. International Journal on Interactive Design and Manufacturing (IJIDeM), $1-21$.

5. Su Jianning, Liu Tingting, \& Wang Peng. Research on Usability Evaluation of Product Modeling Design Based on Naive Bayesian Method. Mechanical Design, V.33; No. 316 (2), 108-111.
6. Lin, L., Guo, Z.E., \& Yang, M.Q. (2020). Current Research Situation and Trend of Product Imagebased Modelling Optimization. Packaging Engineering, 41(02), 65-79.

7. Zhang Fanglan, Yang Minglang, \& Liu Weidong. (2014). Evaluation of Automobile Form Design Scheme Based on Fuzzy Topsis Method. Computer Integrated Manufacturing System (02), 54-61.

8. Tang Bangbei, Guo Gang, Wang Kai, Lin Li, Zhou Jing, \& Fan Xuan, et al. (2015). User Experience Selection of Automobile Industry Design Combined with Eye Movement and EEG. Computer Integrated Manufacturing System, 21 (6).

9. Picton, T. W. (1992). The P300 wave of the human event-related potential. Journal of clinical neurophysiology, 9(4), 456-479.

10. Yang Cheng, Chen Chen, \& Tang Zhichuan. Research on Product Image Reasoning Model Based on EEG. Journal of Mechanical Engineering, 54 (23), 126-136.

11. Wang Qicai, Yu Weidong, Chen Kemin, \& Zhang Zhongwei. (2014). Human Tactile Perception and Brain Cognition of Fabric Stimulation. International Journal of Medical Radiology (4), 323-327. 